Article

\title{
Development of a Piezoelectric Transducer-Based Integrated Structural Health Monitoring System for Impact Monitoring and Impedance Measurement
}

\author{
Ziyi Guo ${ }^{1}$, Tianxiang Huang ${ }^{2, *(1)}$ and Kai-Uwe Schröder ${ }^{2}$ \\ 1 Space Control and Inertial Technology Research Center, Harbin Institute of Technology, \\ Harbin 150001, China; ziyiguo@foxmail.com \\ 2 Institute of Structural Mechanics and Lightweight Design, RWTH Aachen University, Wüllnerstrasse 7, \\ 52062 Aachen, Germany; kai-uwe.schroeder@sla.rwth-aachen.de \\ * Correspondence: tianxiang.huang@sla.rwth-aachen.de
}

Received: 16 February 2020; Accepted: 12 March 2020; Published: 18 March 2020

\begin{abstract}
Structural health monitoring (SHM) techniques, which are also considered as online nondestructive testing methods, are significant in modern structural engineering due to their ability to guarantee structure safety while reducing maintenance cost. It is often necessary to combine different SHM methods to achieve a more reliable damage detection result. However, the hardware of the SHM systems is usually expensive, bulky, and heavy when they are designed separately. Therefore, this paper proposes a three-layer architecture for designing an integrated multi-function SHM system to achieve a small, lightweight, and low power consumption SHM system. Based on the architecture, an integrated SHM system with impact monitoring and electromechanical impedance measurement is developed. In addition, a scheduling module is developed to manage the two functions of the system. Furthermore, an integrated interface is developed to transfer the data and the command. Then, an integrated printed circuit board is designed and manufactured to achieve the aforementioned functions. The designed system is applied for impact monitoring and damage detection for a supporting structure of a sailplane.
\end{abstract}

Keywords: structural health monitoring; piezoelectric transducer; system integration; impact monitoring; electromechanical impedance measurement

\section{Introduction}

With the benefits associated with enhancing safety and reducing maintenance costs, structural health monitoring (SHM) has become an increasingly important topic, especially in the aerospace industry. Unlike conventional nondestructive testing techniques, structural health monitoring refers to the process of continuously monitoring the current state of a structure and measuring damage-sensitive data from the sensors that are typically permanently installed on the structure. Nowadays, many researchers are dedicated to piezoelectric transducer (PZT)-based SHM methods [1-6] because of their performance in damage diagnosis and their stability against noise from the environment. The PZT-based SHM methods can be divided into two categories: the passive method, where sensors are designed to collect data only, and the active method, where the PZT sensors generate vibration or emit stress waves [7].

One of the typical active SHM methods is based on guided waves (GW). These are specific types of elastic waves that are generated and measured by PZTs. This method has been widely used for damage detection in metallic and composite structures because GW can travel a relatively long distance and are sensitive to damage [8-11]. However, components of these kinds of systems consist of a waveform 
generator, power amplifier, charge amplifiers, data acquisition-boards, and computer systems, which make their application to online applications difficult.

Another active sensing technique is based on electromechanical impedance (EMI) $[2,8]$, which can be applied on a composite material [12]. The theoretical model for the EMI method was first established by Liang et al. [13]. In this model, the electrical impedance, which is the inverse of the electrical admittance, of a PZT sensor is shown to be coupled with the mechanical impedance of the host structure. Thus, the measured impendence from PZT can represent the severity of damage in the host structure. However, there are some practical issues related to the application such as debonding issues, temperature effects, and so on [14]. Usually, equipment employed for measuring electric impedance includes an inductance-capacitance-resistance (LCR) meter or impedance analyzer [15]. This hardware, including Agilent 4194A and Wayne Kerr 6500B [16], and so on, is often employed in experiments. Researches using this hardware tend to focus on some practical issues and theoretical problems and pursue a high level of precision; thus, reducing energy consumption and weight of hardware is not considered in these studies. Moreover, some of SHM systems utilizing EMI methods require a set of PZTs to form a sensor network, and this causes the hardware system to become heavy and complex. Therefore, it is necessary to design a lightweight and low-power consumption hardware system for real applications. One way to shrink the size of the hardware system is to employ a chip named AD5933. The AD5933 impedance converter is a high-precision impedance converter system with an on-board frequency generator, a 12-bit 1MSPS, ADC, and an on-board digital signal processing (DSP) engine processing a discrete Fourier transform (DFT) with a weight of less than $5 \mathrm{~g}$. Nowadays, it is employed by many researchers for designing low-cost, on-board, and wireless SHM systems [4]. The next generation of EMI-based system is supposed to be an onboard and sensor system comprising a mini impedance measuring chip such as AD5933 [15].

Conversely, impact detection, which also requires several sensors to form a sensor network, is a typical passive SHM method. Impact detection is usually divided into two missions: impact source location detection and impact energy level detection, and this information can help to conduct maintenance and repair work. Time-of-arrival based on PZT sensor arrays is a common method used for source location [17], whereas wavelet transform can be used to improve the signal-to-noise ratio [18]. Impact energy is also used to perform system identification to calculate the magnitude and location of impacts as studied in [19]. Hardware for impact detection is often related to measuring the charge signal of PZT sensors, which can be lost quickly due to the input resistance of measurement equipment. Therefore, special equipment is necessary. A charge amplifier is a traditional solution for detecting the full impact signal. Liu et al. [20] use a different approach in which they convert the analog impact signal to a digital form where a reference voltage for digitalization is needed. This new design can quickly and steadily process the impact signal, but an accurate method of choosing the reference voltage is still undetermined. In addition, digitalization may cause loss of the impact information. Another solution is a wireless sensor network. One of its advantages is that it can process numerous sensors. Moreover, it can reduce the weight and energy cost of the whole system when there is a significant amount of wire [21]. These hardware systems used to be applied in a library environment, and thus are not suitable for real applications.

To date, researchers [20,22-25] tend to focus on a single SHM method rather than a combination of several methods. However, the integrations of several SHM methods have many advantages. First, the use of different SHM methods can share their merits to provide a completer and more robust SHM system. Second, SHM combinations can also reduce the weight of the total system, and by combining the active and passive SHM methods, the energy consumption can also be reduced. The active method, which requires more energy, will only be active when the passive method detects an issue, and the passive method requires a low level of power consumption and will inspect the structures continuously $[8,26]$.

These advantages have led to many studies that directly combine different SHM methods to achieve a more robust damage detection strategy. Sevillano et al. [27] developed a damage detection 
strategy of combining EMI methods and GW methods. Zheng et al. [28] developed a baseline-free damage detection strategy that combines Lamb waves and EMI methods. An and Sohn [29] designed a system, called the integrated impedance and guided wave (IIG) system, which combines EMI-based and GW-based methods through a baseline-based damage indicator for damage detection. They also proposed a circuit design for the IIG system where a wave generator, a multiplexer (MUX), and a digitizer are involved. However, these only focused on the combination of strategies or only proposed the prototype of the circuit design.

Recently, Islam and Huang [30] achieved damage detection through the combination of the EMI method and GW methods, and Nasrollahi et al. [8] presented an integrated SHM system that includes acoustic emission, EMI, and guided ultrasonic wave. Hyun-Jun Park et al. [31] proposed a wireless hardware scheme for implementing the GW method and measuring EMI signals. The hardware systems involved here are more about the layout of sensors rather than the design of hardware systems and they only utilized disjoint hardware and software parts. Thus, they need to be improved for engineering applications. Nevertheless, limited research has been undertaken to propose a design in which all functions can be integrated into one printed circuit board (PCB).

This paper aims to develop a small, lightweight, low-power consumption PZT-based SHM system which can be applied on real engineering structures. The features of the proposed SHM system are compared with three other systems as illustrated in Table A1 in Appendix A. This system has a three-layer architecture (sensors, hardware, and software). Based on the architecture, an integrated SHM system with impact monitoring and electromechanical impedance measurement is developed. In addition, a scheduling module is developed to manage the two functions of the system. To transfer the data and the command, an integrated interface is developed. Then, an integrated printed circuit board is designed and manufactured to achieve the aforementioned functions. In addition, this article proposes a framework for designing an integrated multi-function SHM system, especially in the management of hardware and software interface, as well as scheduling different damage detection methods.

The rest of the paper is organized as follows. The second section provides a general overview of the entire system. The third section provides the integrated PZT-based hardware system schemes. The corresponding software is described in the fourth section, where the integrated interface is proposed. The experiments on impact detection and damage quantification based on the EMI method are discussed in the fifth section. Finally, conclusions are outlined in the last section, which also suggests the possible future work.

\section{Development Method for an Integrated Piezoelectric Transducer (PZT)-Based SHM}

A significant problem of an integrated PZT-based SHM system is that the different functions need different sampling circuits. Impact monitoring requires several PZT sensors to be sampled simultaneously to locate the place the impact occurs and to evaluate its amplitude. Conversely, the EMI method tends to use one sensor at a time to avoid disturbance from other sensors. This means that the number of PZT sensors should be different for different functions and that there should be different sampling circuits for different usages. Relays serve as channel selectors, but they are bulky and are often used in strong current environments [22]. Note that the multi-channel SHM system works in weak current. Therefore, with the development of microelectronics technology, the MUX has gradually replaced the relay's position in SHM systems [25]. Therefore, a so-called "scheduling module" part, which is formed by several MUXs, is utilized to schedule the commands to the PZT sensors and transfer their signals to different preprocessing units according to the needs. This is the core idea of integrating these two functions mentioned in one PCB. Meanwhile, the corresponding software is required for the controlling and data processing.

Based on this concept, the architecture of the designed SHM system is shown in Figure 1. The system contains three layers: sensor, hardware, and software. Piezoelectric transducers are employed in the sensor layer; the hardware layer includes several parts, such as charge amplifiers, 
$\mathrm{ADC}$, and an impedance converter; and the software layer, also called an integrated interface, plays an important role in integrating these two functions and making the system energy efficient. For instance, the software will normally run the impact monitoring until the impact occurs, and after the impact, the software stops so that the EMI method can run. This procedure would save a lot of energy because it cuts off the running time of EMI method, which is an active sensing and energy consuming method. All the results will show in the guided user interface (GUI) on a computer.

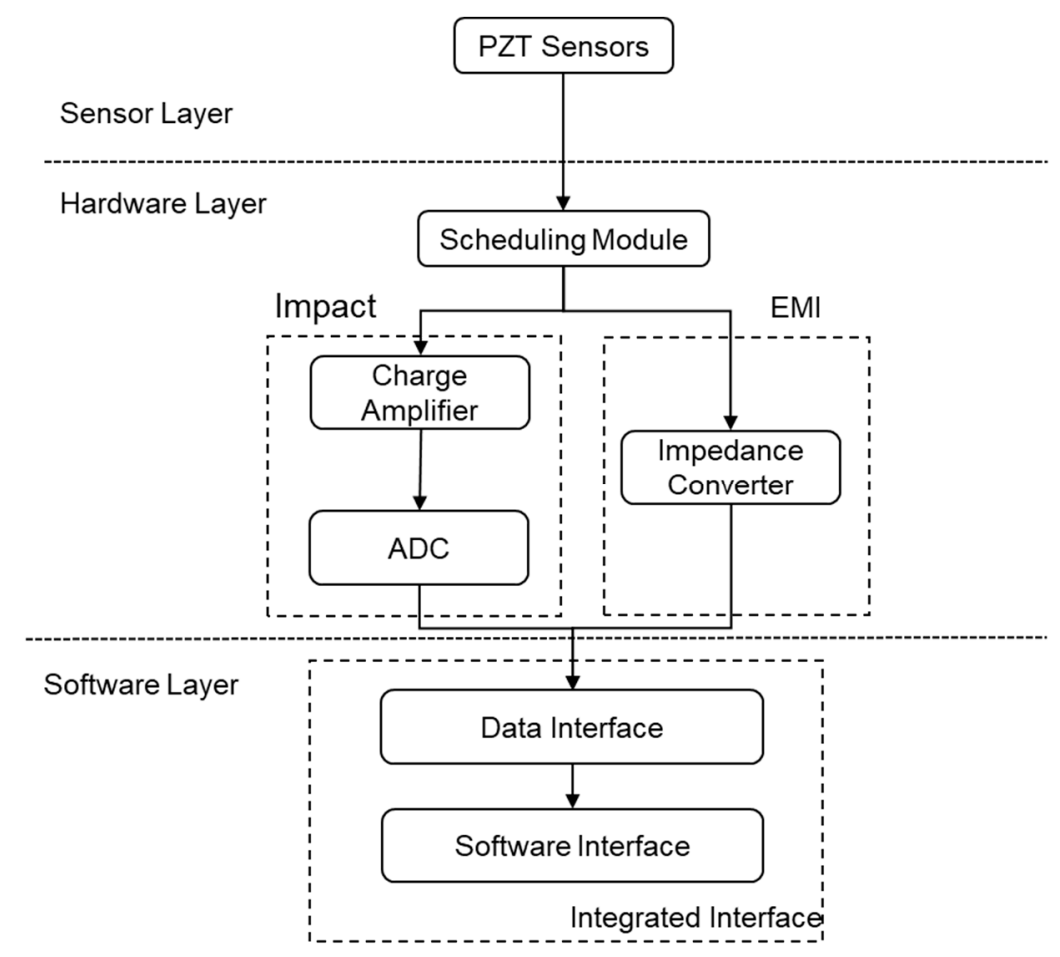

Figure 1. The architecture of the designed structural health monitoring (SHM) system.

The designed system is a multi-channel and multi-function SHM system that can perform impact detection and damage quantification based on the EMI method; these two functions use the same set of PZT sensors. This design not only reduces the number of PZT sensors, but also shrinks the size of the SHM system to make it lightweight and energy efficient, and thus capable of being applied to aerospace structure engineering.

\section{Hardware Development}

In this section, the hardware schemes of the integrated PZT-based system are presented. First, the hardware development of the scheduling module is developed. Then, the scheme for impact detection is proposed. After that, the hardware development for the impedance measurement is proposed. At last, the system realization of the whole hardware system is presented.

\subsection{Scheduling Module on Hardware}

The scheduling module is one of the core components required to achieve the multi-function characteristic of the designed SHM system. As PZT sensors are used as multi-functional sensors, they should be controlled by different circuits for different functions. The basic idea for achieving this is to adopt analog switches. These switches will guide the signal from the PZT sensors to different processing circuit parts according to users' demands.

The architecture of this part is illustrated in Figure 2, and the corresponding scheme is shown in Figure 3. Two analog switches are employed: ADG 752 (Analog Devices Inc., Norwood, MA, USA), a single-pole, double-throw switch, and ADG728 (Analog Devices Inc., Norwood, MA, USA), an 8:1 
matrix switch. These two analog switches are controlled by a microcontroller unit (MCU) through an inter-integrated circuit (IIC) bus as detailed in Figure 3, where the arrowed line indicates the direction of signal flow.

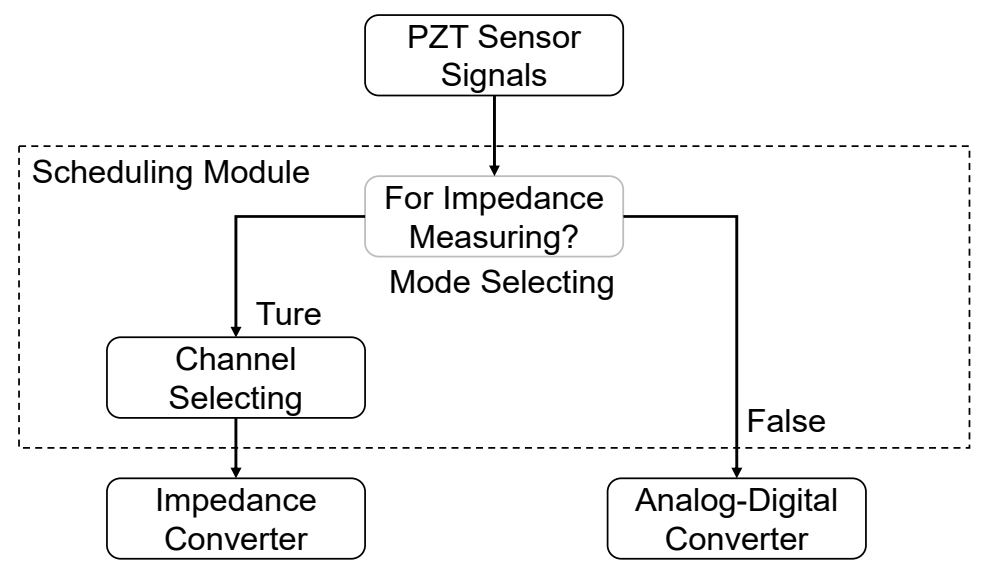

Figure 2. The hardware frame for the scheduling module.

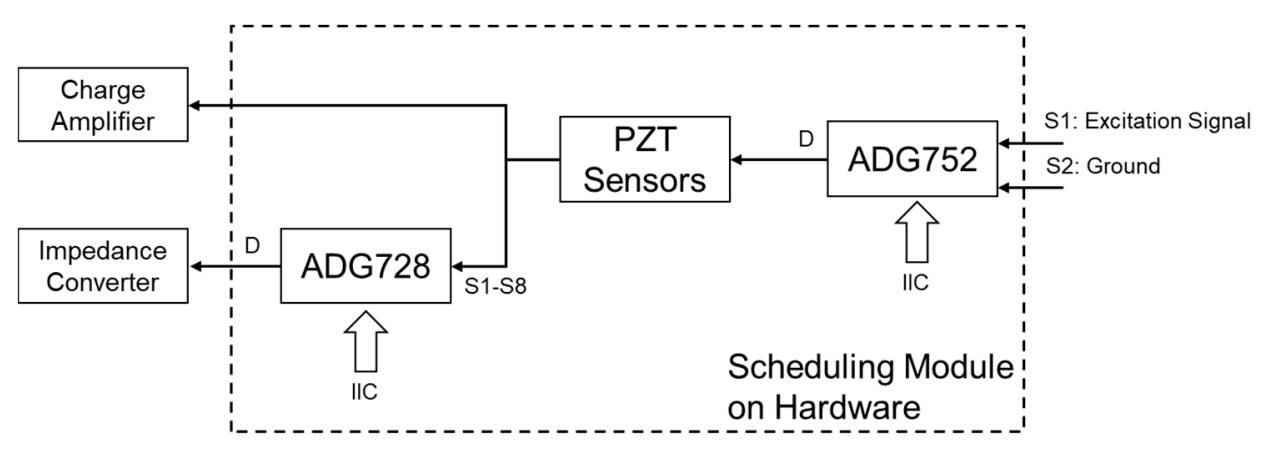

Figure 3. Hardware description of the scheduling module.

The ADG752 in Figure 3 serves as a mode selector. The S1 pin is connected to an impedance converter that generates an excitation signal for impedance measuring mode, while its S2 pin is connected to the ground for impact detecting mode. The microprocessor decides which pin will be connected to the $\mathrm{D}$ pin according to the chosen mode.

If the impact detecting mode is selected, then the ADG752's S2 pin will be connected to the ADG752's D pin; therefore, one of the pins of each PZT sensors is connected to the same ground together, while the other pins are connected to charge amplifiers.

For impedance measuring, the ADG752' S1 pin is connected to that of the D pin. The excitation signal will then flow into the PZT sensors. Next, the microprocessor will control the ADG728 to determine which PZT sensor will undergo impedance measurement by connecting a pin from S1 to $\mathrm{S} 8$ of ADG728 to its D pin. Further signal processing and calculation will be conducted in the impedance converter.

\subsection{Impact Detection}

In many applications, the full waveform of the impact response signal can provide more information about the impact. To keep the full impact information, the charge amplifier rather than the voltage comparator is employed. The essence of that is to measure charges of the PZT sensor. As the charge of PZT sensor tends to be quickly lost due to input resistance, it is necessary to design a charge amplifier that can effectively maintain the charge.

The charge amplifier, also referred to as the first-order integrator, is illustrated in Figure 4. Traditionally, the non-inverting input of a charge amplifier is connected to ground, but in this paper, it 
is connected to a non-zero and constant input to introduce a bias voltage to the output. This design can make the system more robust and universal because it can work over a greater range of power supply. Moreover, this design will not affect the performance of the charge amplifier due to the superposition theorem for electrical circuits. Then, the amplified charge signal will be measured by an analog-to-digital converter (ADC).

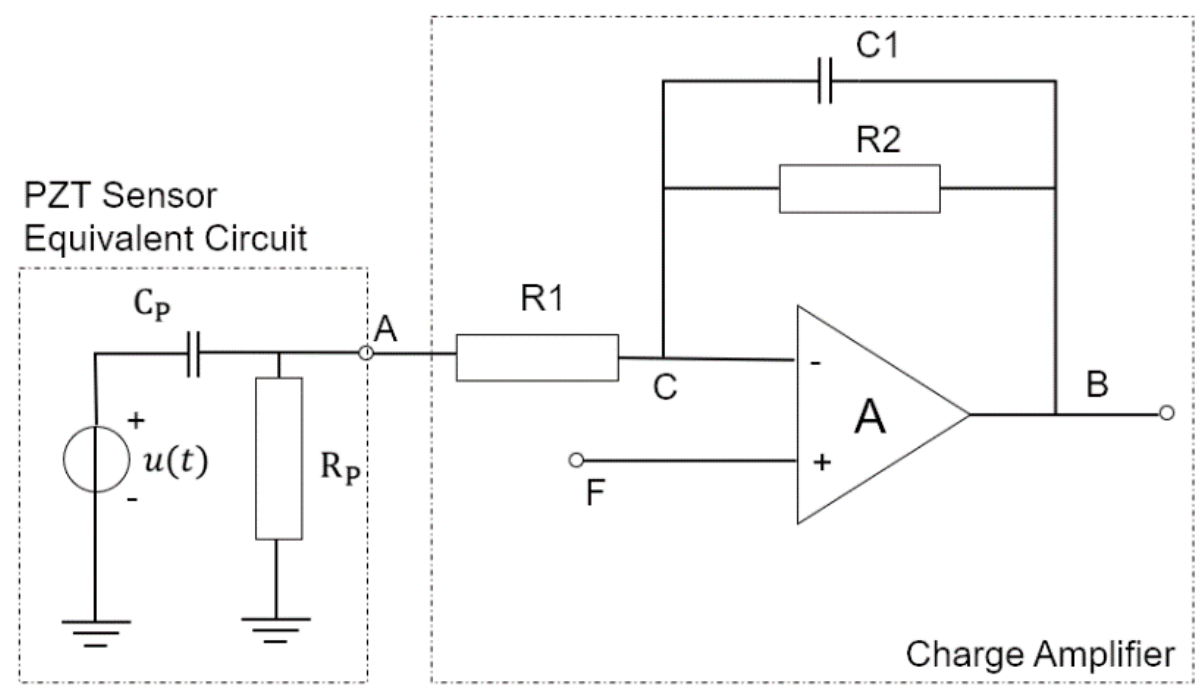

Figure 4. The scheme for the charge amplifier.

\subsection{Impedance Measurement}

In this paper, damage detection is based on the EMI method, which requires a system capable of measuring the electrical impedance of the PZT sensors. To design a lightweight and energy-efficient system, the impedance analyzer, AD5933 (Analog Devices Inc., Norwood, MA, USA), is employed. AD5933 can generate a sinusoidal wave to excite the external impedance and measure the response signal. Then, a DSP in this analyzer can calculate the impedance according to these two signals.

The electrical impedance measurement circuit is based on AD5933, a programmable gain amplifier, and a calibration circuit. This configuration allows a wide measuring range from $100 \Omega$ to $10 \mathrm{M} \Omega$ with a high degree of accuracy. The range is divided into six subranges, as shown in Table 1, and each subrange corresponds to a special feedback resistor of the gain amplifier.

Table 1. The feedback resistors, the calibration resistors, and the corresponding measuring range.

\begin{tabular}{cccc}
\hline Group & Calibration Resistor $(\boldsymbol{\Omega})$ & Gain Resistor $(\boldsymbol{\Omega})$ & Impedance Measuring Range $(\boldsymbol{\Omega})$ \\
\hline 1 & 100 & 100 & $0.1 \mathrm{~K}-1 \mathrm{~K}$ \\
2 & $1 \mathrm{~K}$ & $1 \mathrm{~K}$ & $1 \mathrm{~K}-10 \mathrm{~K}$ \\
3 & $10 \mathrm{~K}$ & $10 \mathrm{~K}$ & $10 \mathrm{~K}-100 \mathrm{~K}$ \\
4 & $100 \mathrm{~K}$ & $100 \mathrm{~K}$ & $100 \mathrm{~K}-1 \mathrm{M}$ \\
5 & 100 & $100 \mathrm{~K}$ & $1 \mathrm{M}-2 \mathrm{M}$ \\
6 & $9 \mathrm{M}$ & $9 \mathrm{M}$ & $>2 \mathrm{M}$ \\
\hline
\end{tabular}

Two modes of the measuring system are illustrated in Figure 5. In the measurement mode, as illustrated in Figure 5a, the excitation signal is first generated by the AD5933, and then goes through a high-pass filter for suppressing the effects of direct current (DC) bias coming from the power supply. Then, the signal is processed by a voltage follower for impedance transformation. However, the voltage follower could introduce high-frequency noises due to a self-oscillation phenomenon. Thus, a low-pass filter (a resistor-capacitor network) is employed to address this issue. The mode selection unit (ADG752, a 2:1 analog switch) guides the signal to a PZT sensor determined by the scheduling module. Then, the response of the PZT, via the programmable gain amplifier (the Signal Amplifier block in Figure 5), 
is measured by AD5933. Finally, the DSP of AD5933 calculates the impedance. The gain resistor of the signal amplifier is selected according to Table 1.

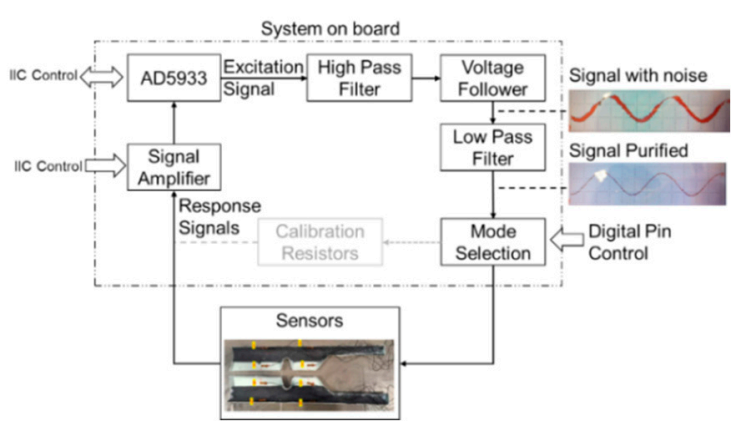

(a)

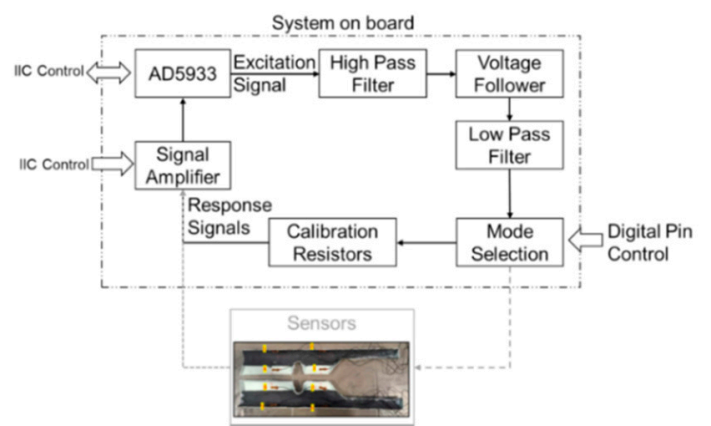

(b)

Figure 5. The circuit principle of the impedance measurement part: (a) Measuring mode: the mode selection part, under the control of the software by digital pin, leads the signal to the sensors. The dotted lines indicate the calibration resistors are not measured; (b) Calibration mode: the mode selection part leads the signal to the calibration resistors to acquire the reference signals. The dotted lines indicate the sensors are not measured.

On the other hand, the calibration mode is illustrated in Figure $5 \mathrm{~b}$. In this mode, the signal from the voltage follower will be guided to a selected calibration resistor by the mode selection unit. Then, the response signal is measured and its impedance is calculated by AD5933. This signal helps the system take the environment changes into account, improving performance. More detailed information on impedance calculation is illustrated in Section 4.3.

\subsection{System Realization}

The hardware is designed and manufactured based on previous sections. It is a lightweight, power-efficient, and eight-channel SHM system, as illustrated in Figure 6. The hardware system has four SMA interfaces on the left and right sides for connecting the eight PZT sensors. There is an additional SMA interface in the upper right corner of the PCB to introduce an external clock signal to the AD5933. This signal is used to drive the AD5933 to measure the impedance of the sensor over $100 \mathrm{kHz}$. In addition, five LEDs are used to indicate the system status: power supply, impedance detection, impact detection, and two IIC communication statuses.

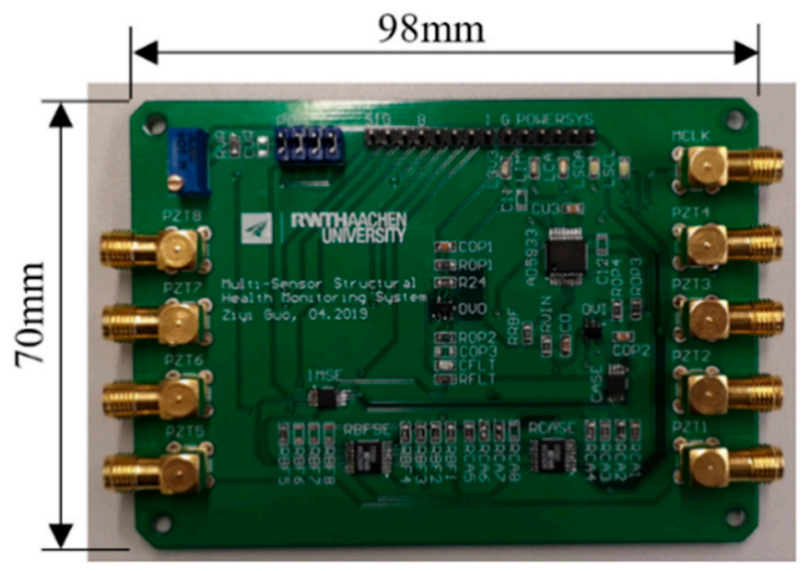

Figure 6. Photo of the designed hardware system.

A double-layer design is adopted in the PCB configuration to reduce the crosstalk. The top layer is used for the EMI, and the bottom layer is for impact detection. Moreover, the components in the 
different layers are designed not to be overlapped. On the other hand, chip resistors and chip capacitors are employed to improve high-frequency performance and resistance to electromagnetic interference. Furthermore, the entire PCB will be protected by an aluminium metal case during service.

The impedance measurement system can measure impedance at the frequency range of $10 \mathrm{~Hz}$ to $100 \mathrm{kHz}$ with a resolution of less than $0.1 \mathrm{~Hz}$. The measurement relative error is less than $0.5 \%$ under the configuration shown in Table 1.

The MCU employed here is STM32F103C8T6 (Shenzhen STS Microelectronics Co. Ltd, Shenzhen, China), whose ADC modules are directly utilized to monitoring the impact signals from charge amplifiers. The ADC is used because it is a 12-bit, 10-channel ADC with a $1 \mathrm{MHz}$ convert rate and direct memory access (DMA) of STM32F103C8T6 can be applied on it. This could improve data read speed and reduce the size of the hardware system. With the power supply voltage of $3.3 \mathrm{~V}$, the maximum error of $\mathrm{ADC}$ is $\pm 4 \mathrm{mV}$ and the average error is $\pm 1 \mathrm{mv}$. The size, power consumption, and other parameters of the system are listed in Table 2.

Table 2. Parameters of the SHM system hardware.

\begin{tabular}{|c|c|c|}
\hline \multicolumn{2}{|c|}{ Parameter } & Value \\
\hline \multicolumn{2}{|c|}{ Size $(\mathrm{mm})$} & $98 \times 70 \times 10$ \\
\hline \multicolumn{2}{|c|}{ Weight (g) } & 125 \\
\hline \multicolumn{2}{|c|}{ Channels } & 8 \\
\hline \multirow{3}{*}{ Power consumption (mW) } & Power Down & $<2$ \\
\hline & Impact Mode & $<66$ \\
\hline & EMI Mode & $<231$ \\
\hline \multicolumn{2}{|c|}{ Power supply (V) } & 3.3 \\
\hline \multicolumn{2}{|c|}{ Frequency range $(\mathrm{Hz})$} & $10-100 \mathrm{k}$ \\
\hline \multicolumn{2}{|c|}{ Impedance measure range $(\Omega)$} & $100-2 \mathrm{M}$ \\
\hline \multicolumn{2}{|c|}{ Frequency Resolution (Hz) } & $0.1 \sim 1 \mathrm{k}$ \\
\hline \multicolumn{2}{|c|}{ Impedance Measurement Error } & $<0.5 \%$ \\
\hline \multicolumn{2}{|c|}{ ADC Measurement Error (mV) } & $<1$ \\
\hline
\end{tabular}

\section{System Software Realization}

\subsection{Integrated Interface and Scheduling Module on Software}

The software system can be considered as an integrated interface that consists of two parts: the software interface and data interface, as illustrated in Figure 7.

The data interface, running on an MCU, drives the underlying hardware, including the impedance analyzer and analog switches. Based on the IIC of the data preprocess block and serial communication, it can deliver the data from the software interface to the hardware and vice versa.

The software interface, running on a computer, mainly handles the subfunctions with the scheduling module, and the results from the scheduling module are processed by the postprocessing module for being ready to display. Finally, the data will be presented to the user on the GUI, which is a human-computer interaction interface and achieves an easy-to-use software system.

More importantly, the integrated interface is also extensible. For example, when the SHM method based on vibration needs to be implemented, only a corresponding block needs to be added in the subfunction block to tell the scheduling module how to handle the new function, instead of redesigning and rewriting the software architecture for the entire upper computer. 


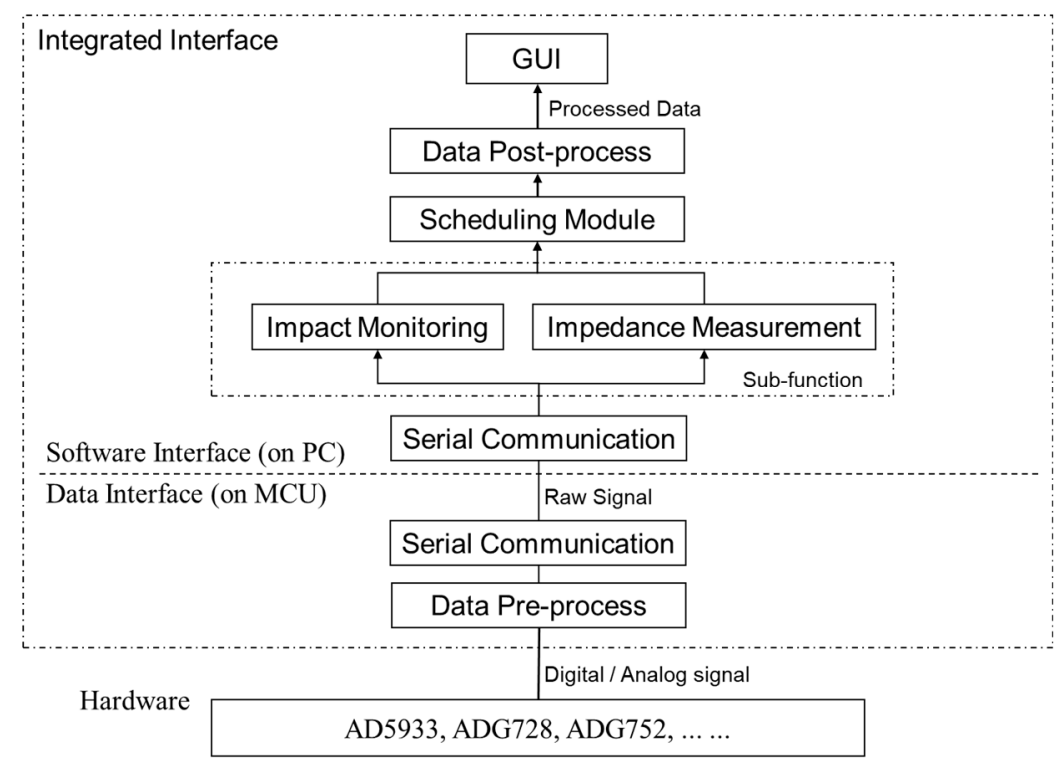

Figure 7. The scheme of the designed integrated interface.

The scheduling module plays a key role in realizing the reasonable collocation of each SHM method. Before running the integrated interface, information of the different subfunctions is programmed into the scheduling model. After the integrated interface starting, this module runs each subfunction according to the information. To be specific, the information for a subfunction contains: (1) whether the subfunction is for impact detecting or damage detecting and (2) when to switch this subfunction to the other subfunction. The first one tells the module how to display the output of this subfunction on the GUI, whereas the second one helps the module form a task chain.

Figure 8 shows how this module works for a typical task chain employed in this study. When the SHM system software is running, the scheduling module first runs the impact monitoring function continuously. If the impact is greater than the preset threshold, the module will stop this function to perform impedance measurements for evaluating the damage. If the damage is detected, then the system will send a message that a repair is needed. If not, the module switch to the impact monitoring function back again. As can be seen, this configuration can avoid running both methods all the time and then save energy.

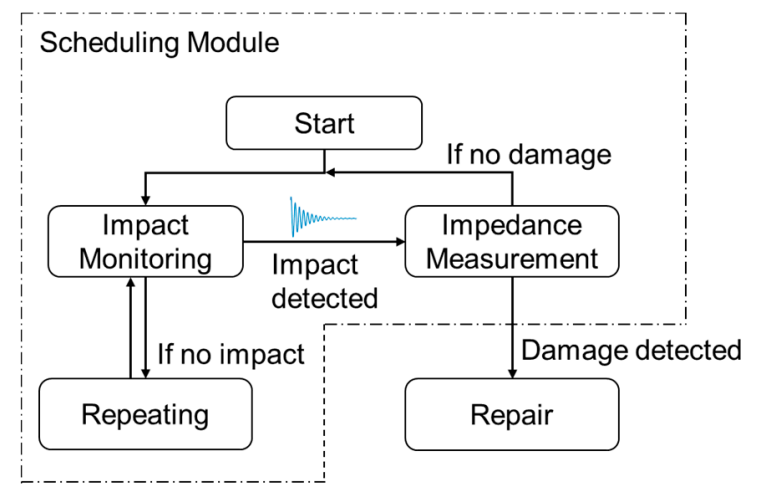

Figure 8. An application example of a scheduling module (on software).

\subsection{Impact Monitoring}

The impact monitoring function is based on the sampling of time-domain signals. After sampling, quantification of the sampling data is launched to check whether an impact over the threshold has occurred. If so, then the system will save the history of this period for further evaluation while sending 
a warning message for the overload. If not, then the sampling will continue as before, until the user stops it. This quantification flow chart is illustrated in Figure 9.

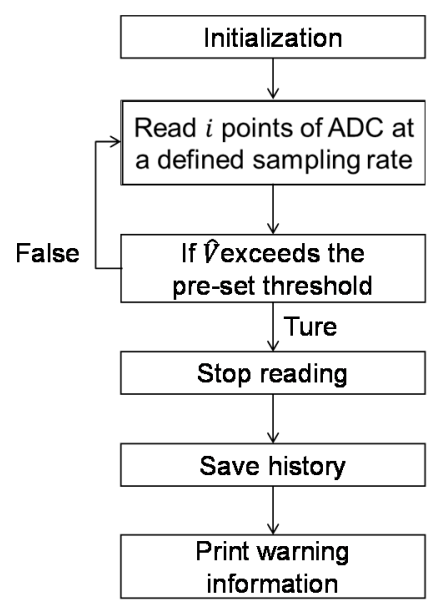

Figure 9. The flow chart of impact monitoring.

The initialization in Figure 9 means that before performing the damage detection function formally, the system will first sample several ADC points of each PZT sensor in a steady state for calibrating. This can improve the robustness of the system because it can adapt to different initial environments.

In this paper, the method based on average voltage deviation is implemented to discover the sensor that is closest to the impact. This can be expressed as

$$
\begin{gathered}
\text { Location }=\underset{i}{\arg \max } \hat{\mathrm{V}}_{\mathrm{i}} \\
\hat{\mathrm{V}}_{\mathrm{i}}=\frac{\left[\sum_{j=1}^{n}\left(V_{i}^{j}-\bar{V}_{i}\right)^{2}\right]^{1 / 2}}{n}
\end{gathered}
$$

where $\hat{V}_{\mathrm{i}}$ is the average voltage deviation of the $i$-th sensor during a certain time period, $V_{j}^{i}$ is the voltage of the ith sensor at time $j$, and $\overline{V_{i}}$ is the average voltage for the period. Note that only discrete sampling points can be obtained; thus, $n$ represents the total sampling points during a sampling period. The right part of Equation (1) will return the sensor number that has the largest signal magnitude during sampling period. Here, location means the place where the impact happened. The $i$-th sensor must have the maximum magnitude among all the sensors attached to the same structure.

\subsection{Impendence Measurement}

One important part of the host software is using the EMI method to quantify the damage to the structure. The flowchart for damage detection with impedance measurement is illustrated in Figure 10. First, choose a resistor group in Table 1 arbitrarily for amplifying the weak response of the PZT sensor and calculating the gain factor. Next, by programming the frequency sweep parameters, including start frequency, frequency increment, and the amount of increments, into AD5933 through serial communication, a sweep cycle begins. The sweep is followed by a calculation of the measured electric impedance. To be specific, the block representing the measuring of the electric impedance in Figure 10 is divided into three steps: 


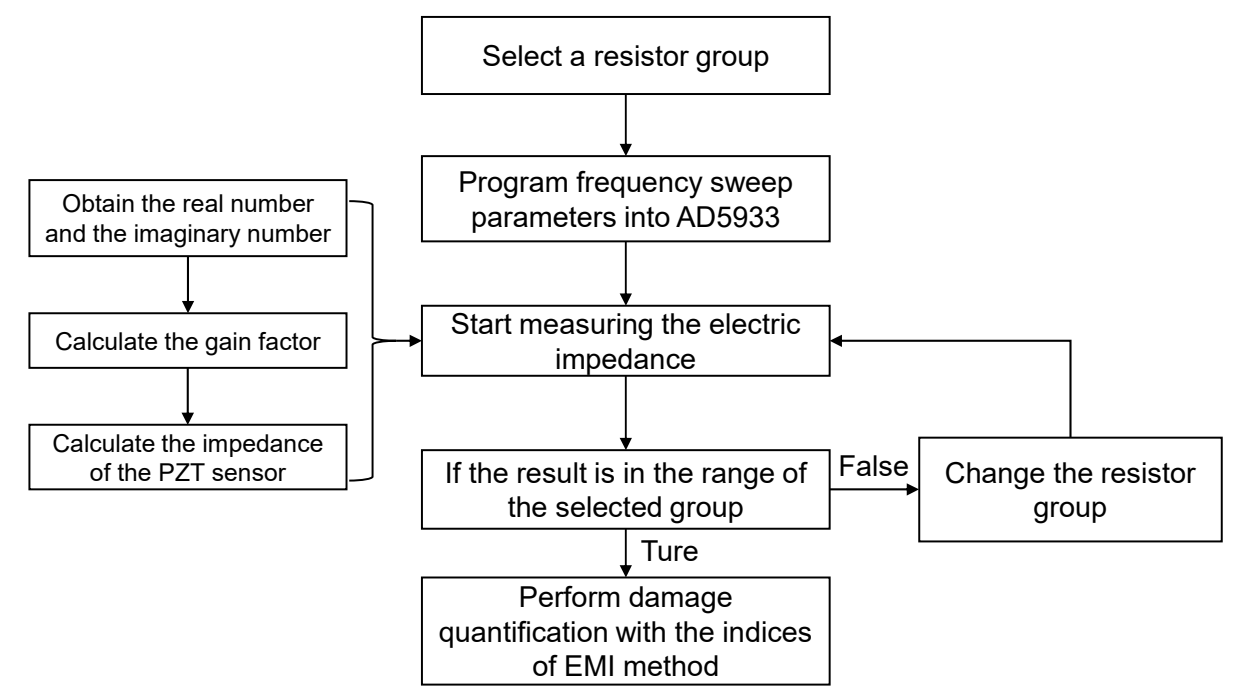

Figure 10. The flowchart of damage detection with impedance measurement.

Step 1: Read the real number $\left(\mathrm{R}_{\mathrm{PZT}}\right)$ and the imaginary number $\left(\mathrm{I}_{\mathrm{PZT}}\right)$ of the PZT sensor via IIC from AD5933.

Step 2: Calculate the gain factor. This means that we first read the real number $\left(\mathrm{R}_{\mathrm{CAL}}\right)$ and the imaginary number $\left(\mathrm{I}_{\mathrm{CAL}}\right)$ of the calibration resistor, and then calculate the magnitude $\left(\mathrm{A}_{\mathrm{CAL}}\right)$ as Equation (3). The impedance of the calibration resistor, which has been already known, is marked as $\mathrm{IM}_{\mathrm{CAL}}$. The gain factor $\mathrm{G}_{\mathrm{CAL}}$ is calculated as Equation (4).

$$
\begin{aligned}
& \mathrm{A}_{\mathrm{CAL}}=\sqrt{\mathrm{R}_{\mathrm{CAL}}^{2}+\mathrm{I}_{\mathrm{CAL}}^{2}} \\
& \mathrm{G}_{\mathrm{CAL}}=\frac{1}{\mathrm{IM}_{\mathrm{CAL}} \times \mathrm{A}_{\mathrm{CAL}}}
\end{aligned}
$$

Step 3: Calculate the impedance of the PZT sensor as

$$
\mathrm{IM}_{\mathrm{PZT}}=\frac{1}{\mathrm{G}_{\mathrm{CAL}} \sqrt{\mathrm{R}_{\mathrm{CAL}}^{2}+\mathrm{I}_{\mathrm{CAL}}^{2}}}
$$

After the impedance calculation, the system should check whether the result is within the range required by the resistor group in Table 1 . If not, then the system needs to change the group and start a new cycle for impedance calculation. Note that Step 2 can be first finished offline to save computational resources.

Several indices are adopted in the system to quantify the damage. Note that the data in the healthy state was already stored in memory before the occurrence of damage. As there is no such a technique that suits every kinds of structure [32], it is necessary to use several indices rather than a single one for damage quantification. The indices employed in the designed system are root mean square deviation (RMSD), mean absolute percentage deviation (MAPD), covariance (Cov), and correlation coefficient (CC) [4]. The RMSD can be expressed as

$$
\operatorname{RMSD}(\%)=\sqrt{\frac{\sum_{i=1}^{n}\left(G_{i}^{1}-G_{i}^{0}\right)^{2}}{\sum_{i=1}^{n}\left(G_{i}^{0}\right)^{2}}}
$$

where $G_{i}^{1}$ is the post-damage conductance at the $i$ point and $G_{i}^{0}$ is the corresponding value in health state. This is a nonparametric statistical damage index due to its computation not involving structure 
parameters. However, in many situations this index cannot differ between the incipient damage and other level of damage clearly.

A variation of the RMSD is MAPD expressed as

$$
\operatorname{MAPD}=\frac{100}{n} \sum_{i=1}^{n} \frac{\left(G_{i}^{1}-G_{i}^{0}\right)}{G_{i}^{0}}
$$

The other two commonly used indicators—Cov and CC—are defined as

$$
\begin{aligned}
\operatorname{Cov}\left(\mathrm{G}^{0}, \mathrm{G}^{1}\right) & =\frac{1}{n} \sum_{i=1}^{n}\left(G_{i}^{0}-\overline{G^{0}}\right)\left(G_{i}^{1}-\overline{G^{1}}\right) \\
C C & =\frac{\operatorname{Cov}\left(\mathrm{G}^{0}, \mathrm{G}^{1}\right)}{\sigma_{0} \sigma_{1}}
\end{aligned}
$$

where $\overline{G^{0}}$ is the average value of the conductance in the health structure; $\overline{G^{1}}$ is the average value of the conductance in the post-damaged structure; and $\sigma_{0}$ and $\sigma_{1}$ are the standard deviations of $\overline{G^{0}}$ and $\overline{G^{1}}$, respectively.

\section{Experimental Validation of the Designed SHM System}

The monitored structure is the main load-bearing structure of a retractable propulsion system for a sailplane, as depicted in Figure 11. It is a cylinder-like structure which is co-cured from two pieces of shell structures. The thickness of the shell structures is $\sim 2 \mathrm{~mm}$. The structure is made from carbon fiber-reinforced plastic and glass fiber-reinforced plastic. Eight encapsulated piezoelectric transducers called PZT layers [33] were adhered to the inner surface of the structure during the manufacturing process, as illustrated in Figure 12. The PIC255 material (Physik Instrumente (PI) GmbH \& Co. KG, Karlsruhe, Germany) was selected as the material of the piezoelectric sensor, and some parameters are shown in the Table 3. The diameter of the PZT ceramic is $8 \mathrm{~mm}$ and the thickness is $0.12 \mathrm{~mm}$. The test for the proposed SHM system is illustrated in Figure 13. Several experiments, including impact amplitude and location detection test and damage detection using EMI methods, are conducted on this subject.

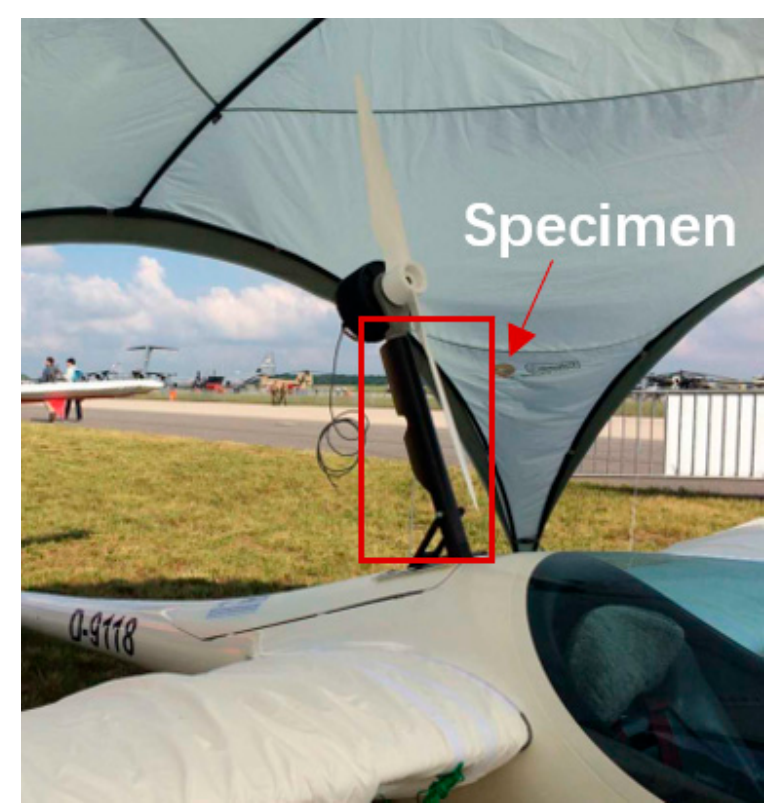

Figure 11. The retractable structure of a sailplane. 


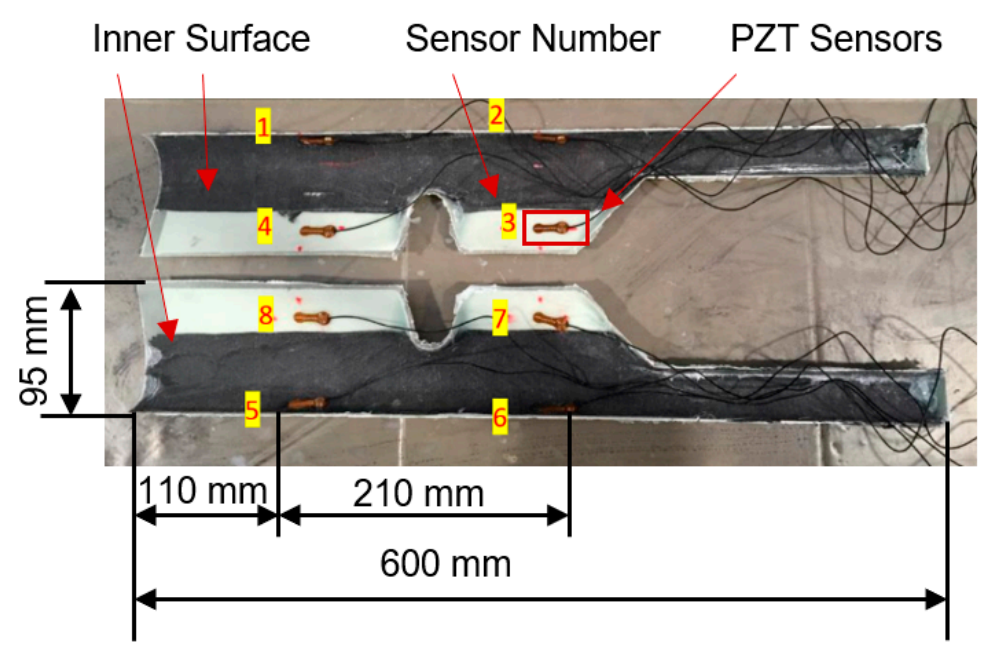

Figure 12. PZT sensors attached to the curved supporting structure.

Table 3. Parameters of the PZT sensor.

\begin{tabular}{cccc}
\hline Parameter & Value & Parameter & Value \\
\hline Density $\left(\mathrm{g} / \mathrm{cm}^{3}\right)$ & 7.80 & $\mathrm{~d}_{31}(\mathrm{pm} / \mathrm{V})$ & -1800 \\
$\varepsilon_{33}^{\mathrm{T}} / \varepsilon_{0}$ & 1750 & $\mathrm{~d}_{33}(\mathrm{pm} / \mathrm{V})$ & 400 \\
$\varepsilon_{11} / \varepsilon_{0}$ & 1650 & $\mathrm{~d}_{15}(\mathrm{pm} / \mathrm{V})$ & 550 \\
Curie Temperature $\left({ }^{\circ} \mathrm{C}\right)$ & 350 & Mechanical Quality Factor $\mathrm{Q}_{\mathrm{m}}$ & 50 \\
\hline
\end{tabular}

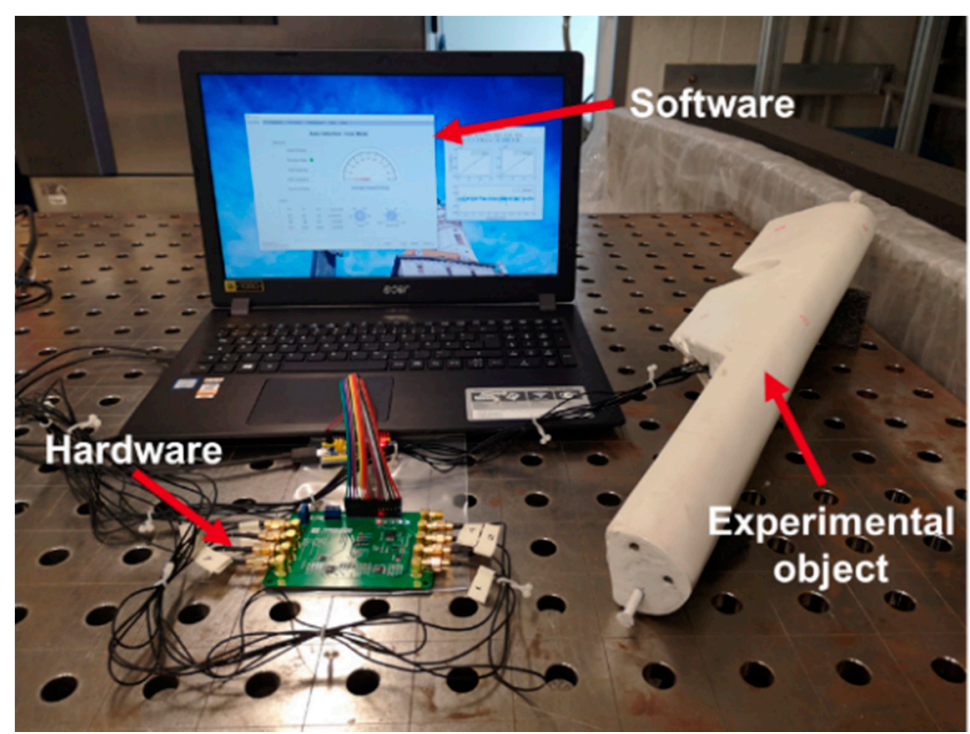

Figure 13. The designed SHM system in experiments.

The aim of this section is to apply impact detection and damage detection using EMI method on the structure shown in Figure 11 to verify the effectiveness of the system. For the system accuracy and other system performance parameters please refer to Table 2 .

\subsection{Impact Amplitude and Location}

An experiment on the impact detection is described in this section. The purpose of this experiment is to verify the impact detection function of the system and verify its ability to distinguish signals from each channel. This ability is used to calculate the impact amplitude and the location of the impact.

The steps for the experiment are as follows. (1) The experimental object is allowed to stand for a certain period of time to ensure the initial state of the system is stable. (2) The initialization switch is 
opened in the designed human-computer interaction interface to complete the initialization process, including setting the sampling frequency at $5 \mathrm{kHz}$ and obtaining the bias voltage applied on ADCs. (3) After the initialization is completed, a hammer is used to introduce impact loads at the area near each sensor (No. 1 to No. 8) sequentially. (4) The response signals corresponding to the eight impact signals are collected. By choosing $n=100$ of Equation (2) as the total sampling points for each period, applying the equation on the sampled impact data, and then selecting the maximum value of each channel during all periods, the results are shown in Table 4. Two typical sets of the time-domain results are revealed in Figures 14 and 15.

Table 4. Maximum voltage of each sensor when strikes are applied to different sensors.

\begin{tabular}{rrcccccccc}
\hline \multirow{2}{*}{$\begin{array}{c}\text { Maximum Voltage } \\
\text { of the Sensor (V) }\end{array}$} & \multicolumn{8}{c}{ Impact Applied at the Sensor (Location) } \\
\cline { 2 - 10 } & No. 1 & No. 2 & No. 3 & No. 4 & No. 5 & No. 6 & No. 7 & No. 8 \\
\hline No. 1 & 1.49 & 0.20 & 0.20 & 0.22 & 0.12 & 0.19 & 0.18 & 0.21 \\
Sensor & No. 2 & 0.61 & 1.61 & 0.55 & 0.29 & 0.39 & 0.26 & 0.93 & 0.75 \\
Number & No. 3 & 0.45 & 0.98 & 1.92 & 0.29 & 0.14 & 0.93 & 1.02 & 0.33 \\
& No. 5 & 0.78 & 0.76 & 0.93 & 1.77 & 0.72 & 0.44 & 0.84 & 0.85 \\
& No. 6 & 0.53 & 0.20 & 0.22 & 0.44 & 1.01 & 0.23 & 0.23 & 1.35 \\
& No. 7 & 0.47 & 0.38 & 0.71 & 0.22 & 0.20 & 1.73 & 0.29 & 0.99 \\
& No. 8 & 0.54 & 0.52 & 0.22 & 0.39 & 0.33 & 0.82 & 0.76 & 0.65 \\
\hline
\end{tabular}
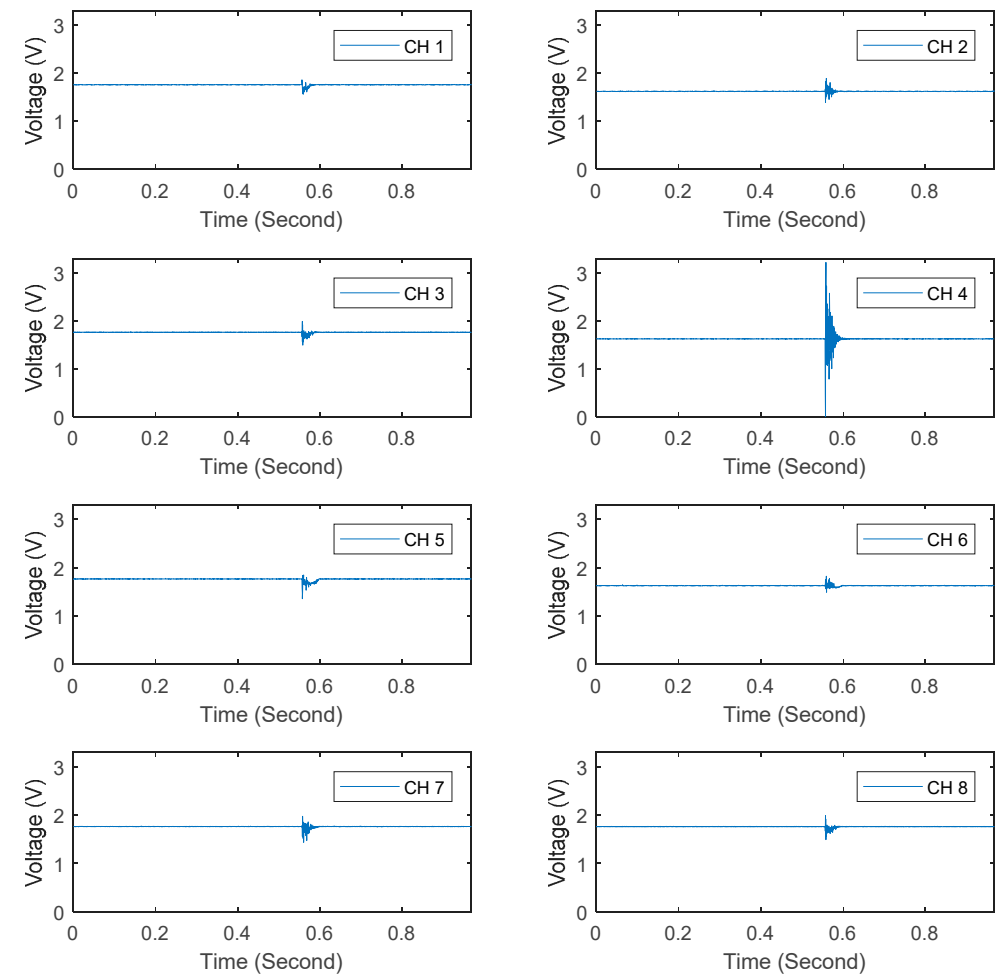

Figure 14. PZT sensor time-domain response signal (impact is introduced near No. 4 sensor). 

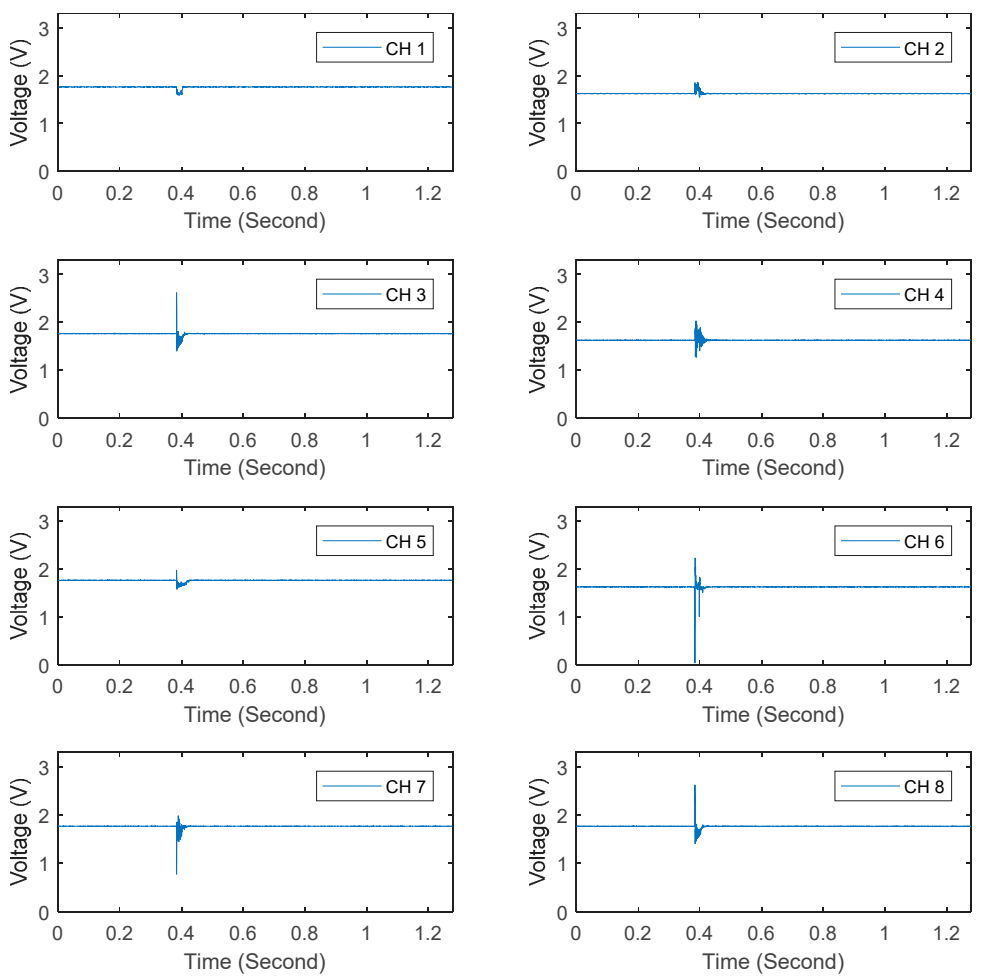

Figure 15. PZT sensor time-domain response signal (impact is introduced near No. 6 sensor).

Figure 15 demonstrates that for each impact signal, the response of the sensor that is closest to the impact signal is most pronounced. It was also found that the response data for the No. 8 sensor showed a random state, and the response to all the impact signals was not obvious. After inspection, it was found that the No. 8 sensor was not functional. The main reason for that might be the poor contact of the interface due to mechanical load during the co-curing and assembling process. The result of this inspection explains this phenomenon. It should be emphasized that as sensor No. 5 is located close to sensor No. 8 in the same half of the structure, when the impact is applied in the area near the sensor location No.8, sensor No. 5 will also receive a relatively large signal.

The time-domain response signals (Figures 14 and 15) also reveal that the designed SHM system can not only distinguish the different impact signals by the sensor responses, but also maintain the original signals from sensors as it is.

To quantify the robustness to measurement noise, the signal-to-noise ratio (SNR) values are calculated. The following SNR definition of the measurement signal is adopted.

$$
\mathrm{SNR}_{\mathrm{dB}}=20 \log \frac{A_{\text {signal }}}{A_{\text {noise }}}
$$

where $A_{\text {signal }}$ represents the peak amplitude of the impact signal and $A_{\text {noise }}$ denotes the peak amplitude of the noise signal.

Based on Equation (10), the SNR of the impact monitoring is $62 \mathrm{~dB}$. The test results indicate that the system has good performance for impact detection in the amplitude evaluation and localization. The system hardware can record the impact response from the sensor, and the system software algorithm can recognize the location of the impact based on these signals.

\subsection{Damage Evaluation Using the EMI Method}

Experiments to verify the EMI method were also conducted. The purpose of experiments was to verify the effectiveness of the EMI method for damage detection, especially to verify whether the system 
can detect the changes in EMI signals of PZT sensors on the irregularly shaped experimental objects, and meanwhile to verify the robustness and repeatability of the hardware system being designed.

The impedance measurement module of the hardware system can measure the impedance with a frequency range from $10 \mathrm{~Hz}$ to $100 \mathrm{kHz}$. Considering that the proposed SHM system is designed for real-time monitoring and with low power consumption, the difference between the upper and lower limits of the measurement frequency range is set to be $5 \mathrm{kHz}$. In addition, based on a pre-experiment, the impedance from $10 \mathrm{kHz}$ to $15 \mathrm{kHz}$ is more sensitive to damage. Thus, the impedance of each PZT sensor is measured at the frequency of from $10 \mathrm{kHz}$ to $15 \mathrm{kHz}$ with a step of $10 \mathrm{~Hz}$, and the excitation signal is in sinusoidal form with an amplitude of $1.98 \mathrm{~V}$. It should be emphasized that for different structures, the range should be changed accordingly, and the designed impedance measurement system can alter the measurement frequency range and the amplitude of excitation signal with the software configuration. Furthermore, only the conductance (the real part of admittance, which is the reciprocal of impedance) was selected for damage detection in the experiment, because the real part of admittance is more robust to the environment than the imaginary part of admittance [34]. In this study, the damage is simulated with different weights of mass blocks. Each mass block weighed $5 \mathrm{~g}$, and it was glued to the structure surface with resin.

Note that in this section, a baseline-based method was used to detect damage. That is, first, the conductance of a PZT sensor in the health state was measured as the baseline data, then the conductance of different damage levels was measured, finally the damage level was evaluated by analyzing the difference of conductance between the damaged one and the health one.

The detailed experiment process is shown below.

(1) Measure the conductance of one PZT sensor in health state, and mark the conductance as ' $\mathrm{C}_{\mathrm{H}}$ '. This conductance is regarded as the baseline (Case 0 ).

(2) Attach a mass block with resin to the structure around the sensor used in step (1), and measure the conductance of the sensor. Mark the result as ' $C_{1}$ '. This conductance is regarded as the data in damage case 1.

(3) Remove the mass block and clean the resin on the surface, then attach the two mass blocks with resin to the structure around the sensor, and measure the conductance of the sensor. Mark the result as ' $\mathrm{C}_{2}$ '. This conductance is regarded as the data in damage case 2.

(4) Remove all the mass blocks, clean the surface. Then, repeat steps (1)-(3) to examine the robustness of the system. Mark these three new conductance lines as $\mathrm{C}_{\mathrm{H}^{\prime}}^{\prime} \mathrm{C}_{1}^{\prime}$, and $\mathrm{C}_{2}^{\prime}$, respectively.

(5) Plot these conductance lines and the difference between the damaged one and health one $\left(\mathrm{C}_{\mathrm{H}}\right)$. Then evaluate these results.

The results are revealed in Figure 16. Figure 16a-c shows the conductance signals in different levels, and Figure 16d,e reveals the effectiveness of baseline method. An analysis of the results is as follows.

(1) Figure 16a-d shows that the conductance signals in the same damage level are almost the same, meaning a good repeatability to the same damage status.

(2) Figure 16e reveals that there is a relatively significant change in conductance signals. Different from Figure 16d, the lines in Figure 16e shift up and two peaks appear. These differences indicate that damage to the structure has occurred.

(3) $C_{2}$ and $C_{2}^{\prime}$ in Figure $16 \mathrm{c}$ are slightly curved compared with $C_{H}$ in Figure 16a. This corresponds to a more significant change of the line, especially of the peak, in Figure 16f compared with Figure 16e, suggesting that more severe damage occurred to the structure. Therefore, the reason for utilizing baseline method is revealed: this method can better reflect the change in conductance to help evaluate the damage level. 

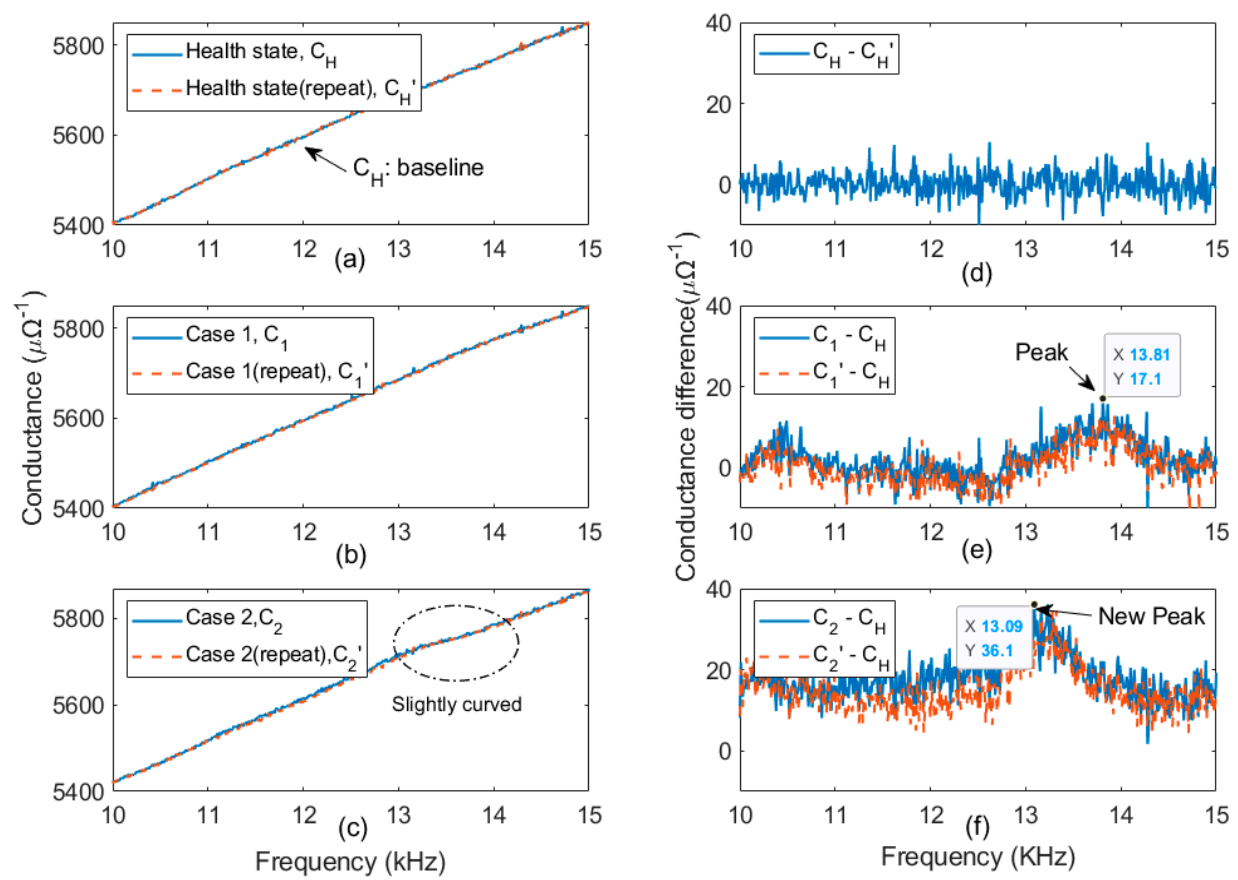

Figure 16. Damage quantification using the electromechanical impedance (EMI) method: (a) the conductance in the health case; (b) the conductance in damage case 1; (c) the conductance in damage case 2; (d) the difference between $\mathrm{C}_{\mathrm{H}}$ and $\mathrm{C}_{\mathrm{H}}^{\prime} ;(\mathbf{e})$ the difference in conductance between damage case 1 and the health case; (f) the difference in conductance between damage case 2 and the health case.

The noise for impendence measurement can be quantified by calculating the SNR of the conductance difference. Based on Equation (10), the SNR of the conductance difference is $20 \mathrm{~dB}$.

To conclude, these results demonstrate that the patterns of conductance of various damage levels are different. Compared with the conductance in health state, the conductance of the structure also changed. The conductance of the same damage level is almost the same, and this demonstrates the repeatability and robustness of the EMI method. The results also verified the effectiveness of the system on the damage detection by applying baseline method on EMI signal.

The indicators of the EMI method introduced in Equations (6)-(9) were applied on the data and the results are listed in Table 5. As can be seen from the table, these indicators can distinguish different damage levels, and the difference between the values of the indicators between the same damage level is also much smaller than that between different damage levels. This means the indicators can well represent the information showing in Figure 16, and show the possibilities of engineering applications.

Table 5. The value of EMI indicators at different damage levels.

\begin{tabular}{ccccc}
\hline Damage & RMSD (\%) & $\mid$ MAPD $\mid$ & $\mid$ Cov $\mid$ & $\mid$ CC $\mid$ \\
\hline Case 0 (repeat) & 0.0729 & $0.709 \times 10^{-4}$ & $5.776 \times 10^{-21}$ & $3.461 \times 10^{-27}$ \\
Case 1 & 0.1286 & $1.232 \times 10^{-4}$ & $4.726 \times 10^{-21}$ & $2.821 \times 10^{-27}$ \\
Case 2 & 0.1658 & $2.506 \times 10^{-4}$ & $0.195 \times 10^{-21}$ & $0.117 \times 10^{-27}$ \\
\hline
\end{tabular}

\section{Conclusions}

In this paper, a multi-channel and multi-function SHM system was developed. The system is lightweight, energy efficient, and can be applied on aerospace structures. This SHM system can perform impact detection and damage quantification based on the EMI method, while both functions use the same set of PZT sensors. The key part of the hardware used to achieve the multi-function characteristic is the scheduling module. 
Experiments were conducted to verify the developed system. In terms of impact detection, the system can record the impact response signal, and, based on this, the system can also effectively identify the location of the impact. In terms of impedance detection, the experiments also confirmed that the electrical impedance changes with the change in the main structure. The indicator method has also proven to be effective on damage detections.

The future topics are the following. The proposed architecture and interface can integrate vibration-based damage assessment. The vibration-based method is based on measurements from accelerometers. Regarding algorithms, introducing a more advanced impact detection method to improve the precision in detecting the impact position and impact amplitude could also be considered. In addition, more studies should be conducted to evaluate the performance of the proposed SHM system under changing environment. Furthermore, the reliability of the whole system through long-term monitoring should be investigated. A wireless system could also be considered for communication and energy harvesting in the next step.

Author Contributions: Conceptualization, T.H.; methodology, T.H.; software, Z.G.; experimental design, Z.G.; validation, Z.G.; writing, original draft preparation, T.H. and Z.G.; writing, review and editing, T.H. and Z.G.; supervision, T.H.; and resources, K.-U.S. All authors have read and agreed to the published version of the manuscript.

Funding: This research was funded by China Scholarship Council grant number 201506830047.

Acknowledgments: The authors gratefully acknowledge the sensor technology support from the Research Center of Structural Health Monitoring and Prognosis and the State Key Laboratory of Mechanics and Control of Mechanical Structures at Nanjing University of Aeronautics and Astronautics. The authors are also grateful to Flugwissenschaftliche Vereinigung Aachen (FVA) for the experimental support. The authors gratefully acknowledge the financial support from the CHINA SCHOLARSHIP COUNCIL for the first author.

Conflicts of Interest: The authors declare no conflict of interest.

\section{Appendix A}

Table A1. Comparison of different structural health monitoring (SHM) systems.

\begin{tabular}{|c|c|c|c|c|c|}
\hline & & $\begin{array}{c}\text { The Proposed SHM } \\
\text { System }\end{array}$ & System 1 [20] & System 2 [27] & System 3 [29] \\
\hline \multirow{3}{*}{$\begin{array}{l}\text { Core hardware } \\
\text { and functions }\end{array}$} & EMI & AD5933 & / & $\begin{array}{l}\text { Impedance analyzer } \\
\text { (HP 4192A) }\end{array}$ & \multirow{2}{*}{$\begin{array}{l}\text { A waveform } \\
\text { generator, two } \\
\text { digitizers and two } \\
\text { multiplexers }\end{array}$} \\
\hline & GW & / & l & $\begin{array}{l}\text { An oscilloscope and } \\
\text { a waveform } \\
\text { generator }\end{array}$ & \\
\hline & $\begin{array}{l}\text { Impact } \\
\text { detection }\end{array}$ & $\mathrm{ADC}$ in STM32F103 & $\begin{array}{l}\text { Comparators } \\
\text { and a field } \\
\text { programmable } \\
\text { gate array }\end{array}$ & / & / \\
\hline \multicolumn{2}{|c|}{ Hardware scheme } & Integrated $\mathrm{PCB}$ & Integrated $\mathrm{PCB}$ & Separated hardware & Separated hardware \\
\hline \multicolumn{2}{|c|}{ Frequency range of EMI } & $10 \mathrm{~Hz} \sim 100 \mathrm{kHz}$ & 1 & $5 \mathrm{~Hz} \sim 13 \mathrm{MHz}$ & I \\
\hline \multicolumn{2}{|c|}{ Cost } & $<\$ 60$ & 1 & $>\$ 3000$ & $>\$ 500$ \\
\hline \multicolumn{2}{|c|}{ Power supply } & $3.3 \mathrm{~V}(<231 \mathrm{~mW})$ & $28 \mathrm{~V}(100 \mathrm{~mW})$ & $\sim 220 \mathrm{~V}$ & $\sim 220 \mathrm{~V}$ \\
\hline \multicolumn{2}{|c|}{ Suitable for online monitoring } & Yes & Yes & No & No \\
\hline
\end{tabular}

\section{References}

1. Annamdas, V.G.M.; Soh, C.K. Application of electromechanical impedance technique for engineering structures: Review and future issues. J. Intell. Mater. Syst. Struct. 2010, 21, 41-59. [CrossRef]

2. Staszewski, W.; Tomlinson, G.; Boller, C.; Tomlinson, G. Health Monitoring of Aerospace Structures; Wiley: Hoboken, NJ, USA, 2004. [CrossRef]

3. Ciang, C.C.; Lee, J.-R.; Bang, H.-J. Structural health monitoring for a wind turbine system: A review of damage detection methods. Meas. Sci. Technol. 2008, 19, 122001. [CrossRef] 
4. Na, W.; Baek, J. A review of the piezoelectric electromechanical impedance based structural health monitoring technique for engineering structures. Sensors 2018, 18, 1307. [CrossRef] [PubMed]

5. Song, G.; Wang, C.; Wang, B. Structural Health Monitoring (SHM) of Civil Structures. Appl. Sci. $2018,789$. [CrossRef]

6. Zhong, Y.; Xiang, J. A two-dimensional plum-blossom sensor array-based multiple signal classification method for impact localization in composite structures. Comput. Aided Civ. Infrastruct. Eng. 2016, 31, 633-643. [CrossRef]

7. Chang, F.K.; Markmiller, J.F.C. A new look in design of intelligent structures with SHM. In Proceedings 3rd European Workshop: Structural Health Monitoring; DEStech Publications, Inc.: Lancaster, PA, USA, 2006.

8. Nasrollahi, A.; Deng, W.; Ma, Z.; Rizzo, P. Multimodal structural health monitoring based on active and passive sensing. Struct. Health Monit. 2018, 17, 395-409. [CrossRef]

9. Rizzo, P.; Marzani, A.; Bruck, J. Ultrasonic guided waves for nondestructive evaluation/structural health monitoring of trusses. Meas. Sci. Technol. 2010, 21, 045701. [CrossRef]

10. Qiu, L.; Yuan, S.; Zhang, X.; Wang, Y. A time reversal focusing based impact imaging method and its evaluation on complex composite structures. Smart Mater. Struct. 2011, 20, 105014. [CrossRef]

11. Qiu, L.; Yuan, S.; Chang, F.-K.; Bao, Q.; Mei, H. On-line updating Gaussian mixture model for aircraft wing spar damage evaluation under time-varying boundary condition. Smart Mater. Struct. 2014, 23, 125001. [CrossRef]

12. Marguerès, P.; Camps, T.; Viargues, M.; Olivier, P. Preliminary experimental study on the electrical impedance analysis for in-situ monitoring of the curing of carbon/epoxy composite material for aeronautical and aerospace structures. Meas. Sci. Technol. 2013, 24, 095005. [CrossRef]

13. Liang, C.; Sun, F.; Rogers, C. An impedance method for dynamic analysis of active material systems. J. Vib. Acoust. 1994, 116, 120-128. [CrossRef]

14. Yang, Y.; Lim, Y.Y.; Soh, C.K. Practical issues related to the application of the electromechanical impedance technique in the structural health monitoring of civil structures: I. Experiment. Smart Mater. Struct. 2008, 17, 035008. [CrossRef]

15. Annamdas, V.G.; Radhika, M.A. Electromechanical impedance of piezoelectric transducers for monitoring metallic and non-metallic structures: A review of wired, wireless and energy-harvesting methods. J. Intell. Mater. Syst. Struct. 2013, 24, 1021-1042. [CrossRef]

16. Zhu, J.; Wang, Y.; Qing, X. A real-time electromechanical impedance-based active monitoring for composite patch bonded repair structure. Compos. Struct. 2019, 212, 513-523. [CrossRef]

17. Ren, Y.; Qiu, L.; Yuan, S.; Su, Z. A diagnostic imaging approach for online characterization of multi-impact in aircraft composite structures based on a scanning spatial-wavenumber filter of guided wave. Mech. Syst. Signal Process. 2017, 90, 44-63. [CrossRef]

18. Ciampa, F.; Meo, M.; Barbieri, E. Impact localization in composite structures of arbitrary cross section. Struct. Health Monit. 2012, 11, 643-655. [CrossRef]

19. Park, J.; Ha, S.; Chang, F.-K. Monitoring impact events using a system-identification method. AIAA J. 2009, 47, 2011-2021. [CrossRef]

20. Liu, P.; Yuan, S.; Qiu, L. Development of a PZT-based wireless digital monitor for composite impact monitoring. Smart Mater. Struct. 2012, 21, 035018. [CrossRef]

21. Yuan, S.; Ren, Y.; Qiu, L.; Mei, H. A multi-response-based wireless impact monitoring network for aircraft composite structures. IEEE Trans. Ind. Electron. 2016, 63, 7712-7722. [CrossRef]

22. Dove, J.R.; Park, G.; Farrar, C.R. Hardware design of hierarchal active-sensing networks for structural health monitoring. Smart Mater. Struct. 2006, 15, 139. [CrossRef]

23. De Castro, B.A.; Baptista, F.G.; Rey, J.A.A.; Ciampa, F. A chromatic technique for structural damage detection under noise effects based on impedance measurements. Meas. Sci. Technol. 2019, 30, 075601. [CrossRef]

24. Qiu, L.; Yuan, S. On development of a multi-channel PZT array scanning system and its evaluating application on UAV wing box. Sens. Actuators A Phys. 2009, 151, 220-230. [CrossRef]

25. Nguyen, K.-D.; Park, J.-H.; Kim, J.-T. Imote2-based multi-channel wireless impedance sensor nodes for local SHM of structural connections. In Sensors and Smart Structures Technologies for Civil, Mechanical, and Aerospace Systems 2011; International Society for Optics and Photonics: San Diego, CA, USA, 2011. [CrossRef]

26. Qing, X.; Li, W.; Wang, Y.; Sun, H. Piezoelectric transducer-based structural health monitoring for aircraft applications. Sensors 2019, 19, 545. [CrossRef] [PubMed] 
27. Sevillano, E.; Sun, R.; Perera, R. Damage detection based on power dissipation measured with PZT sensors through the combination of electro-mechanical impedances and guided waves. Sensors 2016, 16, 639. [CrossRef] [PubMed]

28. Zheng, Y.; Liu, K.; Wu, Z.; Gao, D.; Gorgin, R.; Ma, S.; Lei, Z. Lamb waves and electro-mechanical impedance based damage detection using a mobile PZT transducer set. Ultrasonics 2019, 92, 13-20. [CrossRef] [PubMed]

29. An, Y.-K.; Sohn, H. Integrated impedance and guided wave based damage detection. Mech. Syst. Signal Process. 2012, 28, 50-62. [CrossRef]

30. Islam, M.; Huang, H. Effects of adhesive thickness on the Lamb wave pitch-catch signal using bonded piezoelectric wafer transducers. Smart Mater. Struct. 2016, 25, 085014. [CrossRef]

31. Park, H.-J.; Sohn, H.; Yun, C.-B.; Chung, J.; Lee, M.M. Wireless guided wave and impedance measurement using laser and piezoelectric transducers. Smart Mater. Struct. 2012, 21, 035029. [CrossRef]

32. Soh, C.-K.; Yang, Y.; Bhalla, S. Smart Materials in Structural Health Monitoring, Control and Biomechanics; Springer Science \& Business Media: Berlin/Heidelberg, Germany, 2012. [CrossRef]

33. Qiu, L.; Yuan, S.; Shi, X.; Huang, T. Design of piezoelectric transducer layer with electromagnetic shielding and high connection reliability. Smart Mater. Struct. 2012, 21, 075032. [CrossRef]

34. Park, G.; Kabeya, K.; Cudney, H.H.; Inman, D.J. Impedance-based structural health monitoring for temperature varying applications. JSME Int. J. Ser. A Solid Mech. Mater. Eng. 1999, 42, $249-258$. [CrossRef]

(C) 2020 by the authors. Licensee MDPI, Basel, Switzerland. This article is an open access article distributed under the terms and conditions of the Creative Commons Attribution (CC BY) license (http://creativecommons.org/licenses/by/4.0/). 